USTC-ICTS 03-9

gr-qc/0306044

\title{
Hawking Radiation of an Arbitrarily Accelerating Kinnersley Black Hole: Spin-Acceleration Coupling Effect *
}

\author{
WU Shuang-Qing *, and YAN Mu-Lin † \\ Interdisciplinary Center for Theoretical Study, \& Department of Modern Physics, \\ University of Science and Technology, Hefei 230026, People's Republic of China \\ *E-mail: sqwu@ustc.edu.cn †'E-mail: mlyan@ustc.edu.cn
}

(Dated: revised 17/7, 2003)

\begin{abstract}
The Hawking radiation of Weyl neutrinos in an arbitrarily accelerating Kinnersley black hole is investigated by using a method of the generalized tortoise coordinate transformation. Both the location and temperature of the event horizon depend on the time and on the angles. They coincide with previous results, but the thermal radiation spectrum of massless spinor particles displays a kind of spin-acceleration coupling effect.
\end{abstract}

PACS numbers: 04.70.Dy, 97.60.Lf

In recent ten years, the study of Hawking radiation of some non-stationary black holes, especially with a method of the generalized tortoise coordinate transformation (GTCT), has attracted more attention and achieved much progress. ${ }^{1,2}$ The tortoise coordinate transformation method initially proposed by Damour and Ruffini ${ }^{3}$ to deal with the Hawking effect of scalar fields or Dirac particles in some static spherically symmetric black holes and stationary axisymmetric space-times, ${ }^{4}$ has been generalized by Zhao et al ${ }^{5}$ to the non-static and non-stationary cases and successfully applied to investigate quantum thermal effect of scalar particles in various non-static spherically symmetric space-times and non-stationary black holes. ${ }^{1}$

As far as spinor field case is concerned, the GTCT method has no difficulty in discussing Hawking evaporation of Dirac particles in some non-static spherically symmetric black holes also, ${ }^{1}$ but it is not so easy to use this method to study quantum thermal effect of fermions

\footnotetext{
* Supported partially by China Postdoctoral Science Foundation and K.C. Wong Education Foundation, Hong Kong.
} 
in the non-static and non-spherically symmetric black holes and in the non-stationary black holes. The difficulty lies in the non-separability of Chandrasekhar-Dirac equation ${ }^{6}$ in the most general space-times. Until quite recently this difficulty within the GTCT formalism has been overcome by one of $u^{2}$ via simultaneously treating the first-order and second-order Dirac equations so that one can recast each second-order equation for four spinor components into a standard wave equation near the event horizon. With this extended GTCT method, we first succeed in dealing with the Hawking radiation of Dirac particles in a variable-mass Kerr(-Newman) black hole and observe a new quantum effect called as spin-rotation coupling effect appearing in the thermal radiation spectrum of fermions. ${ }^{7}$ This kind of quantum effect is absent from the bosonic radiation spectrum of scalar particles. Then we reexamine the Hawking evaporation of higher spin particles in the non-static spherically symmetric black holes and show that the Hawking radiation is asymmetric amongst different components of higher spin fields. ${ }^{8}$ Finally we extend this treatment to cope with the Hawking effect of spinor particles in the Kinnersley black hole. ${ }^{9,10}$ Our results show that a spin-acceleration coupling effect displays in the thermal radiation spectrum of Dirac particles in the arbitrarily accelerating Kinnersley black hole ${ }^{10}$ while it does not present in that of fermions in the rectilinearly accelerating Kinnersley black hole. ${ }^{9}$ In all cases we demonstrate that the event horizon equation and Hawking temperature coincide with previously published results.

In this letter, we reinvestigate quantum thermal effect of an arbitrarily accelerating Kinnersley black hole, ${ }^{10}$ but adopt another null tetrad system different from that of Ref. [10]. For simplicity we only consider the case of massless spinor particles namely Weyl neutrinos because the Hawking radiation has of no relation to the mass of particles. ${ }^{2}$ We show that the location of the event horizon and Hawking temperature of the Kinnersley black hole are independent of the choice of a concrete null tetrad system, they coincide with those previously obtained results, however the expression of spin-acceleration effect is much simpler than that obtained in Ref. [10].

The Kinnersley metric ${ }^{11}$ generally called as the "photon rocket" solution, is interpreted as the external gravitational field of an arbitrarily accelerating mass. In the advanced Eddington-Finkelstein coordinate system, the line element of Kinnersley's black hole can be rewritten as

$$
d s^{2}=2 d v(F d v-d r)-r^{2}\left[(d \theta+f d v)^{2}+\sin ^{2} \theta(d \varphi+g d v)^{2}\right]
$$

where $2 F=1-2 M(v) / r-2 a r \cos \theta, f=b \sin \varphi+c \cos \varphi-a \sin \theta$, and $g=(b \cos \varphi-$ $c \sin \varphi) \cot \theta$. The arbitrary function $M(v)$ describes the change in the mass of the source as a function of the advanced time; $a=a(v), b=b(v)$ and $c=c(v)$ are acceleration parameters: $a$ is the magnitude of acceleration, $b$ and $c$ are the rates of change of its direction.

Now we choose such a complex null-tetrad system $\{\mathbf{l}, \mathbf{n}, \mathbf{m}, \overline{\mathbf{m}}\}$ that it satisfies the pseudo- 
orthogonal condition $\mathbf{l} \cdot \mathbf{n}=-\mathbf{m} \cdot \overline{\mathbf{m}}=1$ in which the directional derivatives are

$$
\begin{aligned}
D & =-\partial_{r}, \quad \Delta=\partial_{v}+F \partial_{r}-f \partial_{\theta}-g \partial_{\varphi} \\
\delta & =\frac{1}{\sqrt{2} r}\left(\partial_{\theta}+\frac{i}{\sin \theta} \partial_{\varphi}\right), \quad \bar{\delta}=\frac{1}{\sqrt{2} r}\left(\partial_{\theta}-\frac{i}{\sin \theta} \partial_{\varphi}\right) .
\end{aligned}
$$

It is not difficult to determine the non-vanishing Newman-Penrose complex spin coefficients ${ }^{12}$ in the above null-tetrad as follows [Here and hereafter, we denote $F_{, r}=d F / d r$, etc.]

$$
\begin{aligned}
& \rho=\frac{1}{r}, \quad \mu=\frac{F}{r}-f_{, \theta}, \quad \gamma=\frac{1}{2}\left(-F_{, r}+\frac{i g}{\cos \theta}\right), \\
& \beta=-\alpha=\frac{\cot \theta}{2 \sqrt{2} r}, \quad \nu=\frac{F_{, \theta}}{\sqrt{2} r}=\frac{a \sin \theta}{\sqrt{2}} .
\end{aligned}
$$

In the case of the Kinnersley black hole, $\kappa=\lambda=\sigma=\epsilon=\tau=\pi=0$ and $\psi_{3}=0$, but $\nu \neq 0$, thus it is of Petrov type D. Following Teukolsky's black hole perturbation theory, ${ }^{6}$ one can start from the Weyl neutrino equation

$$
\begin{aligned}
& (D+\epsilon-\rho) \eta_{1}-(\bar{\delta}+\pi-\alpha) \eta_{0}=0 \\
& (\delta+\beta-\tau) \eta_{1}-(\Delta+\mu-\gamma) \eta_{0}=0
\end{aligned}
$$

to derive its corresponding second-order perturbed wave equation

$$
\begin{aligned}
{\left[\left(D+\epsilon^{*}-\rho-\rho^{*}\right)(\Delta+\mu-\gamma)\right.} & \left.-\left(\delta-\alpha^{*}-\tau+\pi^{*}\right)(\bar{\delta}+\pi-\alpha)\right] \eta_{0}=0 \\
{\left[\left(\Delta-\gamma^{*}+\mu+\mu^{*}\right)(D+\epsilon-\rho)\right.} & \left.-\left(\bar{\delta}+\beta^{*}+\pi-\tau^{*}\right)(\delta+\beta-\tau)\right] \eta_{1} \\
& =\left[\nu D+\left(D+2 \epsilon+\epsilon^{*}-\rho^{*}\right) \nu-\psi_{3}\right] \eta_{0} .
\end{aligned}
$$

Write these wave equations (44) and (5) in the above null-tetrad, one obtains their concrete expressions in the Kinnersley black hole

$$
\begin{array}{r}
\left(\partial_{r}+\frac{1}{r}\right) \eta_{1}+\frac{1}{\sqrt{2} r} \mathcal{L}_{1 / 2} \eta_{0}=0 \\
\left(\mathcal{G}-f_{, \theta}-\frac{i g}{2 \cos \theta}\right) \eta_{0}-\frac{1}{\sqrt{2} r} \mathcal{L}_{1 / 2}^{\dagger} \eta_{1}=0
\end{array}
$$

and

$$
\begin{array}{r}
{\left[2 r^{2}\left(\partial_{r}+2 / r\right)\left(\mathcal{G}-f_{, \theta}-\frac{i g}{2 \cos \theta}\right)+\mathcal{L}_{1 / 2}^{\dagger} \mathcal{L}_{1 / 2}\right] \eta_{0}=0} \\
{\left[2 r^{2}\left(\mathcal{G}+F / r-2 f_{, \theta}+\frac{i g}{2 \cos \theta}\right)\left(\partial_{r}+1 / r\right)+\mathcal{L}_{1 / 2} \mathcal{L}_{1 / 2}^{\dagger}\right] \eta_{1}} \\
=\sqrt{2} r^{2} a \sin \theta\left(\partial_{r}+1 / r\right) \eta_{0}
\end{array}
$$

in which the operators $\mathcal{G}, \mathcal{L}_{n}$ and $\mathcal{L}_{n}^{\dagger}$ are defined as

$$
\begin{gathered}
\mathcal{G}=\partial_{v}+F \partial_{r}-f \partial_{\theta}-g \partial_{\varphi}+F / r+F_{, r} / 2 \\
\mathcal{L}_{n}=\partial_{\theta}+n \cot \theta-\frac{i}{\sin \theta} \partial_{\varphi}, \quad \mathcal{L}_{n}^{\dagger}=\partial_{\theta}+n \cot \theta+\frac{i}{\sin \theta} \partial_{\varphi} .
\end{gathered}
$$


After making substitutions $\chi_{1}=\sqrt{2} r \eta_{1}$ and $\chi_{0}=\eta_{0}$, Eqs. ([6) and (17) become

$$
\begin{array}{r}
\partial_{r} \chi_{1}+\mathcal{L}_{1 / 2} \chi_{0}=0 \\
2 r^{2}\left(\mathcal{G}-f_{, \theta}-\frac{i g}{2 \cos \theta}\right) \chi_{0}-\mathcal{L}_{1 / 2}^{\dagger} \chi_{1}=0
\end{array}
$$

and

$$
\begin{aligned}
& {\left[2 r^{2}\left(\partial_{r}+2 / r\right)\left(\mathcal{G}-f_{, \theta}-\frac{i g}{2 \cos \theta}\right)+\mathcal{L}_{1 / 2}^{\dagger} \mathcal{L}_{1 / 2}\right] \chi_{0}=0} \\
& {\left[2 r^{2}\left(\mathcal{G}-2 f_{, \theta}+\frac{i g}{2 \cos \theta}\right) \partial_{r}+\mathcal{L}_{1 / 2} \mathcal{L}_{1 / 2}^{\dagger}\right] \chi_{1}=2 r^{3} a \sin \theta\left(\partial_{r}+1 / r\right) \chi_{0} .}
\end{aligned}
$$

As there exists no Killing vector in an arbitrarily accelerating Kinnersley black hole, one can define the most general form of GTCT as did in Ref. $[1,2,10]$

$$
r_{*}=r+\frac{1}{2 \kappa} \ln \left(r-r_{H}\right), \quad v_{*}=v-v_{0}, \quad \theta_{*}=\theta-\theta_{0}, \quad \varphi_{*}=\varphi-\varphi_{0},
$$

where $r_{H}=r_{H}(v, \theta, \varphi)$ is the location of the event horizon, $\kappa$ is an adjustable parameter and is unchanged under tortoise transformation. All parameters $v_{0}, \theta_{0}$ and $\varphi_{0}$ are arbitrary constants characterizing the initial state of the hole.

Applying the GTCT (10) to the first-order equation (8) and taking the $r \rightarrow r_{H}\left(v_{0}, \theta_{0}, \varphi_{0}\right)$, $v \rightarrow v_{0}, \theta \rightarrow \theta_{0}$ and $\varphi \rightarrow \varphi_{0}$ limits, near the event horizon Eq. (8) reduces to

$$
\begin{aligned}
&\left(r_{H, \theta}+\frac{i}{\sin \theta_{0}} r_{H, \varphi}\right) \frac{\partial}{\partial r_{*}} \chi_{1}+ 2 r_{H}^{2}\left(F-r_{H, v}+f r_{H, \theta}+g r_{H, \varphi}\right) \frac{\partial}{\partial r_{*}} \chi_{0}=0, \\
&-\frac{\partial}{\partial r_{*}} \chi_{1}+\left(r_{H, \theta}-\frac{i}{\sin \theta_{0}} r_{H, \varphi}\right) \frac{\partial}{\partial r_{*}} \chi_{0}=0 .
\end{aligned}
$$

From the vanishing of the determinant of Eq. (11), one gets the following equation that determines the location of event horizon

$$
2\left(F-r_{H, v}+f r_{H, \theta}+2 g r_{H, \varphi}\right)+\frac{r_{H, \theta}^{2}}{r_{H}^{2}}+\frac{r_{H, \varphi}^{2}}{r_{H}^{2} \sin ^{2} \theta_{0}}=0,
$$

which is just the same one derived in Ref. [10]. It should be noted that the location $r_{H}$ we obtained above is just a local point on the section of null surface of the event horizon. This is enough because the GTCT method is a local analysis one. One only needs to calculate the value of the event horizon $r_{H}$ at the specific moment $v_{0}$ and angles $\left(\theta_{0}, \varphi_{0}\right)$. It corresponds to make a analysis locally at every point on the event horizon of a non-stationary black hole (see the last one of Ref. [9] for a complete discussion about this point).

Expand and write out the second-order equation (92) obviously, we have

$$
\begin{aligned}
& \left\{2 r^{2}\left[\partial_{v r}^{2}+F \partial_{r}^{2}-f \partial_{r \theta}^{2}-g \partial_{r \varphi}^{2}\right]+\left(3 r^{2} F_{, r}+6 r F-2 r^{2} f_{, \theta}-r^{2} \frac{i g}{\cos \theta}\right) \partial_{r}\right. \\
& \quad+4 r\left(\partial_{v}-f \partial_{\theta}-g \partial_{\varphi}\right)+\partial_{\theta}^{2}+\frac{1}{\sin ^{2} \theta} \partial_{\varphi}^{2}+\cot \theta \partial_{\theta}+\frac{i \cos \theta}{\sin ^{2} \theta} \partial_{\varphi} \\
& \left.\left.-\frac{1}{4 \sin ^{2} \theta}-\frac{1}{4}+r^{2} F_{, r r}+4 r F_{, r}+2 F-4 r f_{, \theta}-2 r \frac{i g}{\cos \theta}\right)\right\} \chi_{0}=0
\end{aligned}
$$


and

$$
\begin{aligned}
& \left\{2 r^{2}\left[\partial_{v r}^{2}+F \partial_{r}^{2}-f \partial_{r \theta}^{2}-g \partial_{r \varphi}^{2}\right]+\left(r^{2} F_{, r}+2 r F-4 r^{2} f_{, \theta}+2 r^{2} \frac{i g}{\cos \theta}\right) \partial_{r}+\partial_{\theta}^{2}\right. \\
& \left.+\frac{1}{\sin ^{2} \theta} \partial_{\varphi}^{2}+\cot \theta \partial_{\theta}-\frac{i \cos \theta}{\sin ^{2} \theta} \partial_{\varphi}-\frac{1}{4 \sin ^{2} \theta}-\frac{1}{4}\right\} \chi_{1}=2 r^{3} a \sin \theta\left(\partial_{r}+1 / r\right) \chi_{0} .
\end{aligned}
$$

In addition, the Klein-Gordon equation of scalar particles with mass $\mu_{0}:\left(\square+\mu_{0}^{2}\right) \Phi=0$ in the Kinnersley space-time can be written explicitly as

$$
\begin{aligned}
& \left\{2 r^{2}\left[\partial_{v r}^{2}+F \partial_{r}^{2}-f \partial_{r \theta}^{2}-g \partial_{r \varphi}^{2}\right]+\left(2 r^{2} F_{, r}+4 r F-2 r^{2} f_{, \theta}\right) \partial_{r}\right. \\
& \left.\quad+2 r\left(\partial_{v}-f \partial_{\theta}-g \partial_{\varphi}\right)+\partial_{\theta}^{2}+\frac{1}{\sin ^{2} \theta} \partial_{\varphi}^{2}+\cot \theta \partial_{\theta}-\mu_{0}^{2} r^{2}\right\} \Phi=0
\end{aligned}
$$

Now let us consider the asymptotic behaviors of the second-order wave equation near the event horizon. Under the transformation (10), the limiting forms of Eqs. (13),15) are

$$
\begin{aligned}
\tilde{\mathcal{K}} \chi_{0}+[ & -\widetilde{A}+\frac{3 F}{r_{H}}+\frac{3 F_{, r}}{2}-f_{, \theta}-\frac{i g}{2 \cos \theta_{0}}-\frac{i \cos \theta_{0}}{2 r_{H}^{2} \sin ^{2} \theta_{0}} r_{H, \varphi}-\frac{\cot \theta_{0}}{2 r_{H}^{2}} r_{H, \theta} \\
& \left.+\frac{2}{r_{H}}\left(f r_{H, \theta}+g r_{H, \varphi}-r_{H, v}\right)-\left(\frac{r_{H, \theta \theta}}{2 r_{H}^{2}}+\frac{r_{H, \varphi \varphi}}{2 r_{H}^{2} \sin ^{2} \theta_{0}}\right)\right] \frac{\partial}{\partial r_{*}} \chi_{0}=0
\end{aligned}
$$

and

$$
\begin{aligned}
\tilde{\mathcal{K}} \chi_{1}+\left[-\widetilde{A}+\frac{F}{r_{H}}+\right. & \frac{F_{, r}}{2}-2 f_{, \theta}+\frac{i g}{2 \cos \theta_{0}}+\frac{i \cos \theta_{0}}{2 r_{H}^{2} \sin ^{2} \theta_{0}} r_{H, \varphi}-\frac{\cot \theta_{0}}{2 r_{H}^{2}} r_{H, \theta} \\
& \left.-\left(\frac{r_{H, \theta \theta}}{2 r_{H}^{2}}+\frac{r_{H, \varphi \varphi}}{2 r_{H}^{2} \sin ^{2} \theta_{0}}\right)\right] \frac{\partial}{\partial r_{*}} \chi_{1}=r_{H} a \sin \theta_{0} \frac{\partial}{\partial r_{*}} \chi_{0}
\end{aligned}
$$

as well as

$$
\begin{array}{r}
\tilde{\mathcal{K}} \Phi+\left[-\widetilde{A}+\frac{2 F}{r_{H}}+F_{, r}-f_{, \theta}+\frac{1}{r_{H}}\left(f r_{H, \theta}+g r_{H, \varphi}-r_{H, v}\right)\right. \\
\left.-\frac{\cot \theta_{0}}{2 r_{H}^{2}} r_{H, \theta}-\left(\frac{r_{H, \theta \theta}}{2 r_{H}^{2}}+\frac{r_{H, \varphi \varphi}}{2 r_{H}^{2} \sin ^{2} \theta_{0}}\right)\right] \frac{\partial}{\partial r_{*}} \Phi=0
\end{array}
$$

where the operator $\tilde{\mathcal{K}}$ represents the second derivative term

$$
\begin{aligned}
\tilde{\mathcal{K}}= & {\left[\frac{\widetilde{A}}{2 \kappa}+2 F-r_{H, v}+f r_{H, \theta}+g r_{H, \varphi}\right] \frac{\partial^{2}}{\partial r_{*}^{2}}+\frac{\partial^{2}}{\partial r_{*} \partial v_{*}} } \\
& -\left(f+\frac{r_{H, \theta}}{r_{H}^{2}}\right) \frac{\partial^{2}}{\partial r_{*} \partial \theta_{*}}-\left(g+\frac{r_{H, \varphi}}{r_{H}^{2} \sin ^{2} \theta_{0}}\right) \frac{\partial^{2}}{\partial r_{*} \partial \varphi_{*}},
\end{aligned}
$$

and the coefficient $\widetilde{A}$ is an infinite limit of $0 / 0$-type

$$
\begin{aligned}
\widetilde{A} & =\lim _{\substack{r \rightarrow r_{H}, v \rightarrow v_{0} \\
\theta \rightarrow \theta_{0}, \varphi \rightarrow \varphi_{0}}} \frac{F-r_{H, v}+f r_{H, \theta}+g r_{H, \varphi}+r_{H, \theta}^{2} /\left(2 r^{2}\right)+r_{H, \varphi}^{2} /\left(2 r^{2} \sin ^{2} \theta\right)}{r-r_{H}} \\
& =F_{, r}-\left(\frac{r_{H, \theta}^{2}}{r_{H}^{3}}+\frac{r_{H, \varphi}^{2}}{r_{H}^{3} \sin ^{2} \theta_{0}}\right) .
\end{aligned}
$$


Let the coefficient in front of the second-order derivative term $\frac{\partial^{2}}{\partial r_{*}^{2}}$ be $1 / 2$, namely

$$
\frac{\widetilde{A}}{2 \kappa}+2 F-r_{H, v}+f r_{H, \theta}+g r_{H, \varphi} \equiv \frac{1}{2},
$$

then the adjustable temperature parameter $\kappa$ can be determined as

$$
\kappa=\frac{r_{H}^{2} F_{, r}-r_{H}^{-1}\left(r_{H, \theta}^{2}+r_{H, \varphi}^{2} / \sin ^{2} \theta_{0}\right)}{r_{H}^{2}(1-2 F)+\left(r_{H, \theta}^{2}+r_{H, \varphi}^{2} / \sin ^{2} \theta_{0}\right)}
$$

and it coincides with the Hawking temperature derived in Ref. [10].

With such a parameter adjustment, one can recast Eqs. (16]18) into an united standard wave equation near the event horizon

$$
\left[\frac{\partial^{2}}{\partial r_{*}^{2}}+2 \frac{\partial^{2}}{\partial r_{*} \partial v_{*}}-2 C_{3} \frac{\partial^{2}}{\partial r_{*} \partial \theta_{*}}-2 \Omega \frac{\partial^{2}}{\partial r_{*} \partial \varphi_{*}}+2\left(C_{2}+i C_{1}\right) \frac{\partial}{\partial r_{*}}\right] \Psi=0,
$$

where

$$
\Omega=g+\frac{r_{H, \varphi}}{r_{H}^{2} \sin ^{2} \theta_{0}}, \quad C_{3}=f+\frac{r_{H, \theta}}{r_{H}^{2}} .
$$

In the Weyl neutrino case, we have

$$
\begin{aligned}
C_{2}+i C_{1}= & \frac{F}{r_{H}}+\frac{F_{, r}}{2}-f_{, \theta}-\frac{i g}{2 \cos \theta_{0}}-\frac{i \cos \theta_{0}}{2 r_{H}^{2} \sin ^{2} \theta_{0}} r_{H, \varphi} \\
& -\frac{\cot \theta_{0}}{2 r_{H}^{2}} r_{H, \theta}-\frac{1}{2 r_{H}^{2}}\left(r_{H, \theta \theta}+\frac{r_{H, \varphi \varphi}}{\sin ^{2} \theta_{0}}\right), \quad\left(\Psi=\chi_{0}\right), \\
2\left(C_{2}+i C_{1}\right)= & \frac{F}{r_{H}}-\frac{F_{, r}}{2}-2 f_{, \theta}+\frac{i g}{\cos \theta_{0}}+\frac{i \cos \theta_{0}}{2 r_{H}^{2} \sin ^{2} \theta_{0}} r_{H, \varphi} \\
& -\frac{\cot \theta_{0}}{2 r_{H}^{2}} r_{H, \theta}-\frac{1}{2 r_{H}^{2}}\left(r_{H, \theta \theta}+\frac{r_{H, \varphi \varphi}}{\sin ^{2} \theta_{0}}\right) \\
& +\frac{1}{r_{H}^{3}}\left(r_{H, \theta}^{2}+\frac{r_{H, \varphi}^{2}}{\sin ^{2} \theta_{0}}\right)-a r_{H} \sin \theta_{0}\left(r_{H, \theta}-\frac{i r_{H, \varphi}}{\sin \theta_{0}}\right)^{-1}, \quad\left(\Psi=\chi_{1}\right) ;
\end{aligned}
$$

whereas for scalar fields $(\Psi=\Phi)$, we have $C_{1}=0$ and

$$
C_{2}=\frac{F}{r_{H}}-f_{, \theta}-\frac{r_{H, \theta}}{2 r_{H}^{2}} \cot \theta_{0}-\frac{1}{2 r_{H}^{2}}\left(r_{H, \theta \theta}+\frac{r_{H, \varphi \varphi}}{\sin ^{2} \theta_{0}}\right)+\frac{1}{2 r_{H}^{3}}\left(r_{H, \theta}^{2}+\frac{r_{H, \varphi}^{2}}{\sin ^{2} \theta_{0}}\right) .
$$

Following the method of Damour-Ruffini-Sannan's, ${ }^{3}$ the thermal radiation spectra of scalar particles and Weyl neutrinos can be obtained from the standard wave equation (21) near the event horizon ${ }^{10}$

$$
\langle\mathcal{N}(\omega)\rangle \simeq \frac{1}{e^{\left(\omega+m \Omega-C_{1}\right) / T_{H}} \pm 1}, \quad T_{H}=\frac{\kappa}{2 \pi}
$$


in which the coefficient $C_{1}=0$ (for $\left.\Psi=\Phi\right)$; while for $\Psi=\chi_{0}, \chi_{1}$, it reads

$$
\begin{aligned}
C_{1}= & -\frac{g}{2 \cos \theta_{0}}-\frac{r_{H, \varphi} \cos \theta_{0}}{2 r_{H}^{2} \sin ^{2} \theta_{0}}, \quad\left(\Psi=\chi_{0}\right), \\
C_{1}= & \frac{g}{2 \cos \theta_{0}}+\frac{r_{H, \varphi} \cos \theta_{0}}{2 r_{H}^{2} \sin ^{2} \theta_{0}} \\
& -\operatorname{ar}_{H} r_{H, \varphi}\left(r_{H, \theta}^{2}+\frac{r_{H, \varphi}^{2}}{\sin ^{2} \theta_{0}}\right)^{-1}, \quad\left(\Psi=\chi_{1}\right) .
\end{aligned}
$$

It is easy to see that the last term in the right hand side of the second one of Eq. (23) is proportional to the spin coefficient $\nu$. If we neglect this term because it is not invariant under the null tetrad transformation, then the "spin-dependent" term can be written as

$$
\begin{aligned}
\omega_{s} \simeq & s\left(\frac{r_{H, \varphi} \cos \theta_{0}}{r_{H}^{2} \sin ^{2} \theta_{0}}+\frac{g}{\cos \theta_{0}}\right), \\
& \left(s=-1 / 2,1 / 2, \Psi=\chi_{0}, \chi_{1} ; \quad s=0, \Psi=\Phi\right) .
\end{aligned}
$$

The term $\omega_{s}$ reflects that there exists a kind of spin-acceleration coupling effect in the arbitrarily accelerating Kinnersley black hole. ${ }^{2,10}$

In this paper, we have studied the quantum thermal effect of an arbitrarily accelerating Kinnersley black hole. Eqs. (12) and (20) give the location and temperature of the event horizon of the Kinnersley black hole, which depend not only on the advanced time $v$ but also on the angles $\theta$ and $\varphi$. Eq. (22) shows the thermal radiation spectra of scalar particles and Weyl neutrinos in an arbitrarily rectilinearly accelerating Kinnersley black hole. It manifests that there is a new spin-acceleration coupling effect in the arbitrarily accelerating Kinnersley black hole while it disappears in the rectilinearly accelerating Kinnersley black hole. This effect does not present in the thermal radiation spectrum of scalar particles also.

It should be noted that a null tetrad system different from that of Ref. [10] has been chosen here. The difference between them is a Lorentz rotation of type I, however both leads to the same results (the event horizon equation, the Hawking temperature and the thermal radiation spectrum). The expression of the coefficients $C_{1}$ and $C_{2}$ here has a slight difference from that of Ref. [10], it is much simpler than the latter. Besides, both of them have a similar structure, especially the coefficient $C_{1}$ is the same for the $s=-1 / 2$ component. However the coefficients $C_{1}$ and $C_{2}$ for the $s=1 / 2$ component are different in different null tetrad systems, in which there is a term proportional to the spin coefficient $\nu$. The difference probably relates to the shift of the $s=1 / 2$ spinor component under the Lorentz rotation of type I. Thus the term proportional to the spin coefficient $\nu$ can be thought as a gauge dependent term and should be ignored. Though the results have a little difference, the thermal spectrum of spinor particles still reflects qualitatively that there exists a spinacceleration coupling effect in the arbitrarily accelerating Kinnersley black hole. 
In summary, another null tetrad system distinguished from Ref. [10] is chosen to investigate the Hawking effect of the Kinnersley black hole. The choice of basis is more convenient for computations here, and thus the final results are more concise and compact. However we find that the event horizon equation, the Hawking temperature and the thermal radiation spectrum are consistent with those derived in Ref. [10]. We think it should arrive at the same results by choosing different coordinate systems to discuss the Hawking radiation, and conclude that all physical results should be independent of the choice of a concrete coordinate system.

[1] Zhao Z 1999 Thermal Properties of Black Holes and Singularities of Space-times (Beijing Normal University Press, in Chinese)

[2] Wu S Q 2002 Ph. D thesis, Hawking Radiation of Nonstationary Black Holes and Exact Solutions to Scalar Field Equation, Hua-Zhong Normal University (in Chinese)

[3] Damour T and Ruffini R 1976 Phys. Rev. D 14 332; Sannan S 1988 Gen. Rel. Grav. 20239

[4] Liu L and Xu D Y 1980 Acta Phys. Sin. 29 1617; Zhao Z, Gui Y X and Liu L 1981 Acta Astrophys. Sin. 29 141; Xu C M and Shen Y G 1982 Acta Phys. Sin. 31 1035; Xu D Y 1983 Acta Phys. Sin. 32225 (All in Chinese)

[5] Zhao Z and Dai X X 1991 Chin. Phys. Lett. 8 548; Li Z H and Zhao Z 1993 Chin. Phys. Lett. 10126

[6] Chandrasekhar S 1983 The Mathematical Theory of Black Holes (New York: Oxford University Press); Carmeli M 1982 Classical Fields: General Relativity and Gauge Theory (John Wiley \& Sons, New York)

[7] Wu S Q and Cai X 2001 Chin. Phys. Lett. 18 485; 2001 Gen. Rel. Grav. 33 1181; 2002 Gen. Rel. Grav. 34557 and 605

[8] Wu S Q and Cai X 2001 Int. J. Theor. Phys. 40 1349; 2001 Mod. Phys. Lett. A16 1549; 2001 IL Nuovo Cimento B116 907; 2002 Int. J. Theor. Phys. 41559

[9] Wu S Q and Cai X 2002 Chin. Phys. Lett. 19 141; 2002 Int. J. Theor. Phys. 41 641; 2002 Chin. Phys. 11 661; Wu S Q, Zeng Y, Cai X and Yan M L 2003 Acta Phys. Sin. 521340

[10] Wu S Q and Cai X 2002 Gen. Rel. Grav. 341207

[11] Kinnersley W 1969 Phys. Rev. 1861335

[12] Newman E T and Penrose R 1962 J. Math. Phys. 3566 